\begin{tabular}{l}
\hline OPEN OACCESS Freely available online \\
http://www.banglajol.info/index.php/BJID/index \\
Correspondence \\
Bangladesh Journal of Infectious Diseases \\
April 2020, Volume 7, Number Suppl_1, Page S45-S47 \\
ISSN (Online) 2411-670X \\
ISSN (Print) 2411-4820 \\
DOI: https://doi.org/10.3329/bjid.v7i0.46801
\end{tabular}

\title{
Mental Health and Psychosocial Support (MHPSS) in COVID-19 Outbreak
}

\author{
MM Jalal Uddin ${ }^{1}$, Mohammad Tariqul Alam², Helal Uddin Ahmed ${ }^{3}$, Niaz Mohammad Khan ${ }^{4}$, \\ Surazit Kumar Talukder ${ }^{5}$
}

\begin{abstract}
${ }^{1}$ Associate Professor, Department of Psychiatry, National Institute of Neurosciences and Hospital, Dhaka, Bangladesh; ${ }^{2}$ Associate Professor, Department of Psychiatry, National Institute of Mental Health, Dhaka, Bangladesh; ${ }^{3}$ Associate Professor, Department of Psychiatry, National Institute of Mental Health, Dhaka, Bangladesh; ${ }^{4}$ Associate Professor, Department of Psychiatry, National Institute of Mental Health, Dhaka, Bangladesh; ${ }^{5}$ Assistant Professor, Department of Psychiatry, National Institute of Neurosciences and Hospital, Dhaka, Bangladesh
\end{abstract}

[Received: 26 March 2020; Accepted: 2 April 2020; Published: 25 April 2020]

\section{TO THE EDITORS,}

WHO and public health authorities around the globe are taking action to contain the COVID-19 outbreak. However, this time of crisis is generating stress among the people. These mental health considerations were developed by the Mental Health Department as support for mental and psychological well-being during COVID-19 outbreak.

The Inter Agency Standing Committee (IASC) used the term 'mental health and psychosocial support' (MHPSS) in Emergency Settings to describe 'any type of local or outside support that aims to protect or promote psychosocial well-being and/or prevent or treat mental health condition'. The global humanitarian system uses the term MHPSS to unite a broad range of actors responding to emergencies such as the COVID-19 outbreak. The IASC Guidelines for MHPSS in Emergency Settings recommends that multiple levels of interventions be integrated within outbreak response activities. These levels align with a spectrum of mental health and psychosocial needs and are represented in a pyramid of interventions (Figure I) ranging from embedding social and cultural considerations in basic services, to providing specialized services for individuals with more severe conditions. Core principles include: do no harm, promote human rights and equality, use participatory approaches, build on existing resources and capacities, adopt multi-layered interventions and work with integrated support systems.

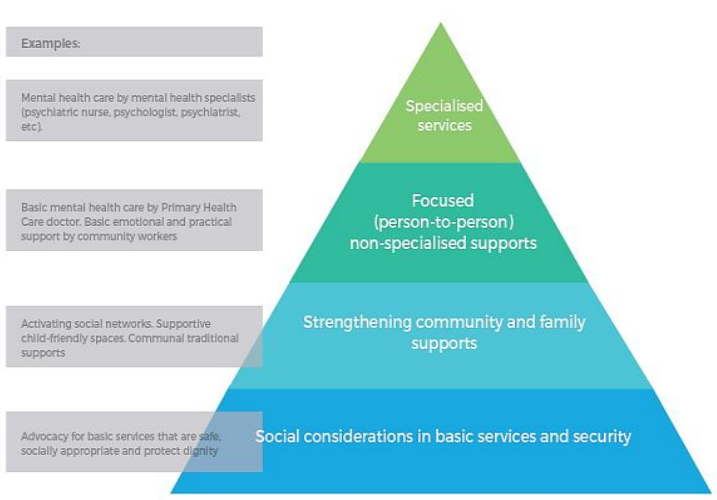

Figure I: Intervention pyramid for mental health and psychosocial support

In any epidemic, it is common for individuals to feel stressed and worried. Common responses of people affected (both directly and indirectly) might include; fear of ill and dying, losing livelihoods, quarantine, separated from loved ones and caregivers. Other than fear avoiding approaching health facilities and refusal to care for separated minors. Any emergency is stressful, but some specific stressors like risk of being infected and infecting others, common symptoms of other infection can be mistaken for COVID-19, worried for children, risk of physical and mental health of special population like older adults, disabled people are particular to COVID-19 affect people. Persons who are directly involve to the care of COVID-19 affected experience additional stressor during outbreak. Constant fear, worry, uncertainties and stressors in population during COVID-19 outbreak 
can lead to long term consequences within families, vulnerable individuals and communities. Among the consequences deterioration of social network, local dynamics, economics, stigma towards surviving patients resulting in rejection by communities, anger, aggression, mistrust, sleep disturbance, developing or aggravating mental health and substance abuse disorders. On the other hand some people may have positive experiences, such as pride about finding ways of coping and resilience. Community members often show great altruism and cooperation, and people may experience great satisfaction from helping others.

MHPSS approaches need to evolve and adapt to the needs of each population affected by COVID-19 and at different times of the outbreak. (i.e. before, during and after high infection rates).

Preparedness will considerably improve and accelerate the response at the onset of an outbreak. Countries where the epidemic hasn't yet spread need to prepare a possible MHPSS response. Such countries should use the current response to the COVID-19 outbreak, and MHPSS work done during previous outbreaks, to guide their preparation. Understanding and addressing mental health and psychosocial considerations will be key to stopping trans-mission and preventing the risk of long-term repercussion on the population's wellbeing and capacity to cope with adversity. This includes the integration of MHPSS approaches and activities within community strategies, community outreach, case identification and contact tracing, as well as activities at health facilities and quarantine sites.Mental health interventions should be carried out within general health services (including primary health care (PHC)) and could in addition be organized in other pre-existing structures in the community, such as schools, community centers, youth and senior centers. The mental health and wellbeing of frontline workers needs to be addressed and supported. Healthcare workers, case identifiers, workers involved in dead body management, and many other staff and volunteers need to be provided with ongoing MHPSS both during and after the outbreak.

Globally recommended some activities key activities that should be implemented as part of the response to COVID-19 are rapid assessment of the context and of culturally specific MHPSS issues, needs and available re-sources; strengthen MHPSS coordination by facilitating collaboration between MHPSS agencies, government and other partners; use information from gender sensitive assessments, including identified needs, gaps and existing resources, to set up/contribute to a system for the identification and provision of care to people with common and severe mental health conditions and substance abuse disorders; establish a MHPSS strategy for COVID-19 cases, survivors, contacts, family members, frontline workers and the broader community, with special attention to the needs of special or/and vulnerable groups ; integrate mental health and psychosocial considerations into all response activities; ensure that accurate information about COVID-19 is readily available and accessible to frontline workers; patients infected with COVID19 , as well as community members. train all frontline; referral pathway for persons with mental health conditions is activated between all sectors involved; develop activity toolkits that parents, teachers and families can use with their children in isolation, including messages on preventing the spread of the disease such as hand washing games $\&$ rhymes; establish opportunities for the bereaved to mourn in a way that does not compromise public health strategies to reduce the spread of COVID-19 but reflects the traditions and rituals of the community; establish measures to reduce the negative impact of social isolation in quarantine sites. in the early recovery phase, support health authorities to establish sustainable and communitybased mental health and psychosocial services and establish monitoring, evaluation, accountability and learning mechanisms to measure effective MHPSS activities.

Social stigma and discrimination can be associated with COVID-19, including towards persons who have infected, their family members and health care and other frontline workers. Steps must be taken to address stigma and discrimination at all phases of COVID-19 emergency response.

There are several interventions recommended by IASC.

Intervention 1: Helping older adults cope with stress during the COVID-19 outbreak

Intervention 2: Supporting the needs of people with disabilities during COVID-19 outbreak

Intervention 3: Messages and activities for helping children deal with stress during the COVID-19 outbreak

Intervention 4: MHPSS activities for adults in isolation/quarantine

Intervention 5: Supporting people working in the COVID-19 response

Intervention 6: Community MHPSS messages during the COVID-19 outbreak 


\section{References}

1. World Health Organization (WHO). Mental Health Consideration during COVID-19 Outbreak. WHO, 2020.

2. IASC Interim Briefing Notes Addressing Mental Health and Psychosocial Aspects of COVID-19 Outbreak. Version 1.5

3. Inter-Agency Standing Committee (IASC). IASC Guidelines on Mental Health and Psychosocial Support in Emergency Settings. IASC: Geneva, 2007.

4. IASC Reference Group on Mental Health and Psychosocial Support in Emergency Settings. Mental Health and Psychosocial Support in Ebola Virus Disease Outbreaks: A Guide for Public Health Programme Planners. IASC: Geneva, 2015

[Bangladesh Journal of Infectious Diseases, April 2020;7(suppl_1):S45-S47]
Correspondence: Dr. MM Jalal Uddin, Associate Professor, Department of Psychiatry, National Institute of Neurosciences and Hospital, Sher E Bangla, Agargaon, Dhaka 1207, Bangladesh; Email: jalal29march@yahoo.com; Cell no.: $+8801716772020$

Conflict of interest: All authors have no competing interests. How to cite this article: Uddin MMJ, Alam MT, Ahmed HU, Khan NM, Talukder SK. Mental Health and Psychosocial Support (MHPSS) in COVID-19 Outbreak. Bangladesh J Infect Dis 2020;7(suppl_1):S45-S47

Copyright: (2020. Uddin et al. Published by Bangladesh Journal of Infectious Diseases. This article is published under the Creative Commons CC BY-NC License (https://creativecommons.org/licenses/by-nc/4.0/). This license permits use, distribution and reproduction in any medium, provided the original work is properly cited, and is not used for commercial purposes. 